\title{
Interference of stimulated electronic Raman scattering and linear absorption in coherent control
}

\author{
J. Rioux, ${ }^{1}$ J. E. Sipe, ${ }^{2}$ and Guido Burkard ${ }^{1}$ \\ ${ }^{1}$ Department of Physics, University of Konstanz, D-78457 Konstanz, Germany \\ ${ }^{2}$ Department of Physics and Institute for Optical Sciences, \\ University of Toronto, 60 St. George Street, Toronto, Ontario, Canada M5S 1A7
}

(Dated: September 22, 2014)

\begin{abstract}
We consider quantum interference effects in carrier and photocurrent excitation in graphene using coherent electromagnetic field components at frequencies $\omega$ and $2 \omega$. The response of the material at the fundamental frequency $\omega$ is presented, and it is shown that one-photon absorption at $\omega$ interferes with stimulated electronic Raman scattering (combined $2 \omega$ absorption and $\omega$ emission) to result in a net contribution to the current injection. This interference occurs with a net energy absorption of $\hbar \omega$ and exists in addition to the previously studied interference occurring with a net energy absorption of $2 \hbar \omega$ under the same irradiation conditions. Due to the absence of a bandgap and the possibility to block photon absorption by tuning the Fermi level, graphene is the perfect material to study this contribution. We calculate the polarization dependence of this all-optical effect for intrinsic graphene and show that the combined response of the material at both $\omega$ and $2 \omega$ leads to an anisotropic photocurrent injection, whereas the magnitude of the injection current in doped graphene, when transitions at $\omega$ are Pauli blocked, is isotropic. By considering the contribution to coherent current control from stimulated electronic Raman scattering, we find that graphene offers tunable, polarization sensitive applications. Coherent control due to the interference of stimulated electronic Raman scattering and linear absorption is relevant not only for graphene but also for narrow-gap semiconductors, topological insulators, and metals.
\end{abstract}

PACS numbers: 73.50.Pz, 42.65.Dr, 78.67.Wj, 42.65.-k

\section{INTRODUCTION}

Coherent control (CC) involves the interference between multiple excitation pathways to acquire a handle on the final state of a quantum-mechanical process. ${ }^{1}$ The field originates from progress in manipulating the transition rates of multiphoton molecular processes ${ }^{2,3}$ and has since grown to encompass condensed-matter systems, including bulk and nanostructured semiconducting materials, ${ }^{4-9}$ metal-semiconductor heterostructures, ${ }^{10}$ and optical lattices. ${ }^{11}$ Applied to semiconductor optics, the typical CC experiment uses light at a fundamental frequency $\omega$ and its second harmonic $2 \omega$. Interference between the transition amplitudes for two-photon absorption (2PA) at $\omega$ and one-photon absorption (1PA) at $2 \omega$ results in an unbalanced distribution of carriers in momentum space, providing a net photocurrent. ${ }^{4,5}$ The two-color electromagnetic field provides the energy necessary to create electronhole pairs in the sample, and the injected charge carriers acquire their velocities according to the band dispersion. ${ }^{6}$

In the typical operation regime for conventional semiconductors such as GaAs, $\hbar \omega$ would lie within the bandgap; the light frequencies in such experiments are regularly selected according to $\hbar \omega<E_{g}<2 \hbar \omega$, where $E_{g}$ is the bandgap energy, so that 1PA at $\omega$ is energetically forbidden, and the lowest energetically-allowed perturbative order for absorption of the fundamental is the second order. However, for $\hbar \omega>E_{g}$, the fundamental is absorbed at first order, and how this affects the CC is especially important to understand for narrow-gap semiconductors. Gapless materials such as graphene further present the possibility of studying $\mathrm{CC}$ in interesting ways by substituting the bandgap $E_{g}$ with $2|\mu|$, where $\mu$ is the chemical potential. The range of applicability of previous theories can thus be handily tested by varying the doping level or an applied gate voltage, and scenarios lying outside the typi- cal operation regime are readily accessible. In particular, for $\hbar \omega>2|\mu|$, linear absorption of the fundamental beam occurs, which has not been carefully considered in early studies of coherent optical injection and control in carbon nanotubes, ${ }^{12-14}$ graphene, ${ }^{15-18}$ and other semiconducting materials. ${ }^{1,4-9}$

In the presence of coherent $\omega$ and $2 \omega$ fields, linear absorption at $\omega$ is not the only process with a net energy absorption of $\hbar \omega$; at the next perturbative order the combined absorption of a $2 \omega$ photon and the emission of a $\omega$ photon must also be considered. This nondegenerate two-photon transition process is an instance of stimulated electronic Raman scattering (ERS), ${ }^{19-21}$ where light is scattered inelastically and energy is deposited into the electronic state of the system, and was recently studied in the nonlinear optical response of graphene ${ }^{22}$ and topological insulators. ${ }^{23}$ Since ERS at $\omega$ has the same initial and final state as 1PA at $\omega$, quantum interference occurs between these two transition pathways. Moreover, since the typical CC term between 1PA and 2PA occurs at $2 \omega$ rather than $\omega$, there are in effect two distinct interference channels.

In this paper, we consider the carrier response to a twocolor field due to the ERS transition amplitude, first generally by presenting a microscopic expression derived from Fermi's golden rule applicable to condensed-matter systems, then specifically for the case of graphene. For linearly-polarized light, it is shown that current injection in graphene becomes anisotropic with respect to the angle between the linear polarization axes of the light when the additional ERS contribution is considered, and that this polarization sensitivity can be tuned by adjusting the chemical potential of the sample. The current injection resulting from the quantum interference between 1PA and ERS is five times stronger with perpendicular polarization axes compared to parallel polarization axes of the light. In contrast, the typical interference term between $1 \mathrm{PA}$ and $2 \mathrm{PA}$ in graphene results in a current magnitude in- 
dependent of the polarization of the light. ${ }^{16}$ The polarization sensitivity is adjustable, via the chemical potential, between a completely isotropic current, to a strongly anisotropic current response where the two contributions to current injection are balanced to exactly cancel each other under perpendicular orientation of the polarization axes.

We derive the microscopic form of the response tensors for carrier and photocurrent injection via quantum interference of 1PA and ERS in Sec. II. We consider the case of graphene, explicitly calculate the response tensors, and present a complete picture of two-color coherent current control via quantum interference of one- and two-photon processes in this material in Sec. III. Finally, we conclude in Sec. IV.

\section{TWO-COLOR INTERFERENCE AT $\hbar \omega$}

In the presence of a two-color optical field with frequency components $\omega$ and $2 \omega$, the conventional injection current as originally described by Atanasov et al. ${ }^{5}$ stems from the crossterm of the following transition amplitudes connecting the same initial and final states: $\Omega_{c v}^{(1)}(2 \omega ; \mathbf{k})$, resulting from light at $2 \omega$ to first order in perturbation, and $\Omega_{c v}^{(2)}(\omega ; \mathbf{k})$, resulting from light at $\omega$ to second order in perturbation. Here $\Omega_{c v}^{(\ell)}(\omega ; \mathbf{k})$ is the degenerate $\ell$-photon transition amplitude between valence band $v$ and conduction band $c$ at wavevector $\mathbf{k}^{24}$ The one- and two-photon absorption processes are illustrated by their Feynman diagrams in Fig. 1(a).

The injection term for the current density that results from the coherent interference between the 1PA and 2PA transition amplitudes has the form

$$
j^{a}=\eta_{I}^{a b c d}(\omega) E^{b *}(\omega) E^{c *}(\omega) E^{d}(2 \omega)+\text { c.c. }
$$

where $\eta_{I}(\omega)$ is a fourth-rank tensor describing the current response of the material, $\mathbf{E}(\omega)[\mathbf{E}(2 \omega)]$ is the electric field component at frequency $\omega[2 \omega]$, and repeated Roman superscripts are summed over Cartesian directions. The tensor is given explicitly for a two-dimensional material by:

$$
\begin{aligned}
\eta_{I}^{a b c d}(\omega)=\frac{\pi i e^{4}}{\hbar^{3} \omega^{3}} \sum_{c, v} & \int \frac{\mathrm{d}^{2} k}{4 \pi^{2}}\left[v_{c c}^{a}(\mathbf{k})-v_{v v}^{a}(\mathbf{k})\right] \\
& \times w_{c v}^{b c *}(\mathbf{k}) v_{c v}^{d}(\mathbf{k}) \delta\left[\omega_{c v}(\mathbf{k})-2 \omega\right]
\end{aligned}
$$

where $e=-|e|$ is the electron charge,

$$
w_{c v}^{b c}(\mathbf{k}) \equiv \sum_{m} \frac{v_{c m}^{b}(\mathbf{k}) v_{m v}^{c}(\mathbf{k})+v_{c m}^{c}(\mathbf{k}) v_{m v}^{b}(\mathbf{k})}{\omega_{m c}(\mathbf{k})+\omega_{m v}(\mathbf{k})},
$$

$\mathbf{v}_{m n}(\mathbf{k}) \boldsymbol{\delta}\left(\mathbf{k}-\mathbf{k}^{\prime}\right)=\left\langle m \mathbf{k}|\mathbf{v}| n \mathbf{k}^{\prime}\right\rangle$ indicate matrix elements of the velocity operator $\mathbf{v}, \omega_{c v}(\mathbf{k}) \equiv \omega_{c}(\mathbf{k})-\omega_{v}(\mathbf{k})$, and $\hbar \omega_{m}(\mathbf{k})$ are the band energies. ${ }^{5,16}$

There is however another contribution to the current of the form of Eq. (1) that vanishes in a semiconductor when typical frequencies are chosen to lie within the bandgap. This additional term stems from interference between $\Omega_{c v}^{(1)}(\omega ; \mathbf{k})$ describing one-photon absorption at $\omega$ and $\Omega_{c v}^{(2)}(2 \omega,-\omega ; \mathbf{k})$ (a)

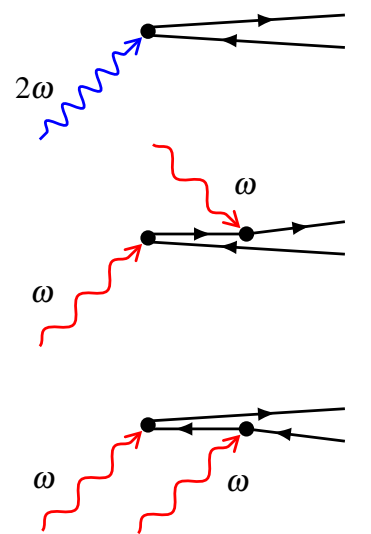

(b)

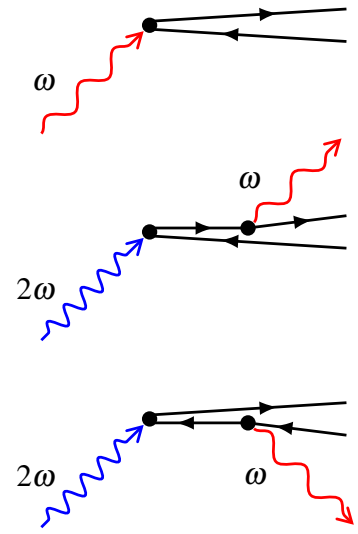

Figure 1. Feynman diagrams of first- and second-order absorption processes contributing to coherent control in the presence of a twocolor, $\omega$ and $2 \omega$ field. The time axis points to the right, a plain arrow pointing to the right (left) represents an electron (hole), and vertices represent the dipole interaction. (a) Processes where the net energy absorption amounts to $2 \hbar \omega$ : one-photon absorption (1PA) at $2 \omega$ and two-photon absorption (2PA) at $\omega$; the final state is an electron-hole pair of energy $2 \hbar \omega$. (b) Processes where the net energy absorption amounts to $\hbar \omega$ : 1PA at $\omega$ and stimulated electronic Raman scattering (ERS) at $\omega(2 \omega$ absorption and $\omega$ emission); the final state is an electron-hole pair of energy $\hbar \omega$. The crossed processes, where light is emitted before absorption, are also included in Eq. (5) but their diagrams omitted for brevity. Second-order processes where the net energy absorption amounts to $3 \hbar \omega$ or $4 \hbar \omega$ are not included since they do not contribute to coherent control unless third- and higher-order absorption processes are also included.

describing the nondegenerate two-photon process of absorption at $2 \omega$ and emission at $\omega$. These processes are illustrated by three Feynman diagrams in Fig. 1(b). The first diagram shows the one-photon absorption process; a $\omega$ photon is absorbed and an electron-hole pair is created with energy $\hbar \omega$. This process has a k-dependent transition amplitude ${ }^{24}$ given by

$$
\Omega_{c v}^{(1)}(\omega ; \mathbf{k})=\frac{i e}{\hbar \omega} v_{c v}^{a}(\mathbf{k}) E^{a}(\omega)
$$

and leads to the well-known linear absorption of $\omega$ light. The second and third diagrams of Fig. 1(b) show the nondegenerate two-photon process; a $2 \omega$ photon is absorbed, a virtual electron-hole pair is created with energy $2 \hbar \omega$, and a $\omega$ photon is emitted by either the electron (second diagram) or the hole (third diagram), reducing the energy of the electron-hole pair to $\hbar \omega$. This is an instance of a stimulated electronic StokesRaman process ${ }^{19-21}$ known to induce a two-color optical Kerr effect. $^{25}$ We calculate the $\mathbf{k}$-dependent transition amplitude in the single-particle approximation at the level of Fermi's golden rule, and find

$$
\Omega_{c v}^{(2)}(2 \omega,-\omega ; \mathbf{k})=\frac{e^{2}}{\hbar^{2} \omega^{2}} w_{c v}^{\prime a b}(\mathbf{k}) E^{a *}(\omega) E^{b}(2 \omega),
$$


where

$w_{c v}^{\prime a b}(\mathbf{k}) \equiv \sum_{m}\left(\frac{v_{c m}^{a}(\mathbf{k}) v_{m v}^{b}(\mathbf{k})}{\omega_{m}(\mathbf{k})-\left[\omega_{c}(\mathbf{k})+\omega\right]}+\frac{v_{c m}^{b}(\mathbf{k}) v_{m v}^{a}(\mathbf{k})}{\omega_{m}(\mathbf{k})-\left[\omega_{v}(\mathbf{k})-\omega\right]}\right)$.

The first term within the summation in Eq. (6) corresponds to the second diagram of Fig. 1(b), the latter term corresponds to the third diagram.

The injection rate for the expectation value of any operator $\Theta$ is then calculated up to second perturbative order from $\langle\dot{\Theta}\rangle=i \hbar^{-1}\langle[H, \Theta]\rangle$ using matrix elements of the Hamiltonian given by $H_{m n}(\mathbf{k})=\hbar \omega_{m}(\mathbf{k}) \delta_{m n}-\frac{e}{c} \mathbf{v}_{m n}(\mathbf{k}) \cdot \mathbf{A}(t)$, where $\mathbf{A}(t)$ is the vector potential, to obtain

$$
\begin{aligned}
\langle\dot{\Theta}\rangle= & 2 \pi \sum_{c, v} \int \frac{\mathrm{d}^{2} k}{4 \pi^{2}}\langle c, v, \mathbf{k}|\Theta| c, v, \mathbf{k}\rangle \\
\times & \left(\left|\Omega_{c v}^{(1)}(\omega ; \mathbf{k})+\Omega_{c v}^{(2)}(2 \omega,-\omega ; \mathbf{k})\right|^{2} \delta\left[\omega_{c v}(\mathbf{k})-\omega\right]\right. \\
& \left.+\left|\Omega_{c v}^{(1)}(2 \omega ; \mathbf{k})+\Omega_{c v}^{(2)}(\omega ; \mathbf{k})\right|^{2} \delta\left[\omega_{c v}(\mathbf{k})-2 \omega\right]\right) .
\end{aligned}
$$

The two summands within brackets describe electron-hole pairs of energy $\hbar \omega$ and $2 \hbar \omega$, respectively. The latter yields the typical CC term described by Eq. (2) that has been the subject of previous studies; we thus focus on the former. Taking the occupation number operator as $\Theta$ yields the injection rate for electron-hole pair creation at $\hbar \omega$,

$$
\begin{aligned}
\dot{n}(\omega) & =\xi_{1}^{a b}(\omega) E^{a *}(\omega) E^{b}(\omega) \\
& +\left[\xi_{I}^{\prime a b c}(\omega) E^{a *}(\omega) E^{b *}(\omega) E^{c}(2 \omega)+\text { c.c. }\right] \\
& +\xi_{2}^{a b c d}(2 \omega,-\omega) E^{a *}(2 \omega) E^{b *}(\omega) E^{c}(2 \omega) E^{d}(\omega) .
\end{aligned}
$$

The first term is one-photon carrier injection due to 1PA, the third term is nondegenerate, two-photon carrier injection (at $\omega)$ due to ERS, and the second term is their interference. The quantities $\xi_{1}, \xi_{I}^{\prime}$ and $\xi_{2}$ are second-, third- and fourth-rank tensors describing the material part of the response. The 1PA carrier injection tensor is described elsewhere. ${ }^{5}$ The response tensor describing the interference is given by

$$
\xi_{I}^{\prime a b c}(\omega)=\frac{2 \pi i e^{3}}{\hbar^{3} \omega^{3}} \sum_{c, v} \int \frac{\mathrm{d}^{2} k}{4 \pi^{2}} v_{c v}^{a *}(\mathbf{k}) w_{c v}^{\prime b c}(\mathbf{k}) \delta\left[\omega_{c v}(\mathbf{k})-\omega\right],
$$

and the ERS carrier injection tensor is given by

$$
\begin{array}{r}
\xi_{2}^{a b c d}(2 \omega,-\omega)=\frac{2 \pi e^{4}}{\hbar^{4} \omega^{4}} \sum_{c, v} \int \frac{\mathrm{d}^{2} k}{4 \pi^{2}} w_{c v}^{\prime d a *}(\mathbf{k}) w_{c v}^{\prime b c}(\mathbf{k}) \\
\times \delta\left[\omega_{c v}(\mathbf{k})-\omega\right] .
\end{array}
$$

The interference term is analogous to coherent population control of electron-hole pairs with net energy absorption of $2 \hbar \omega$ in the conventional CC regime and is nonzero for noncentrosymmetric materials. ${ }^{26}$

Finally, taking the current operator as $\Theta$, the response tensor describing the current injection resulting from interference between ERS and 1PA at $\omega$ is found to be

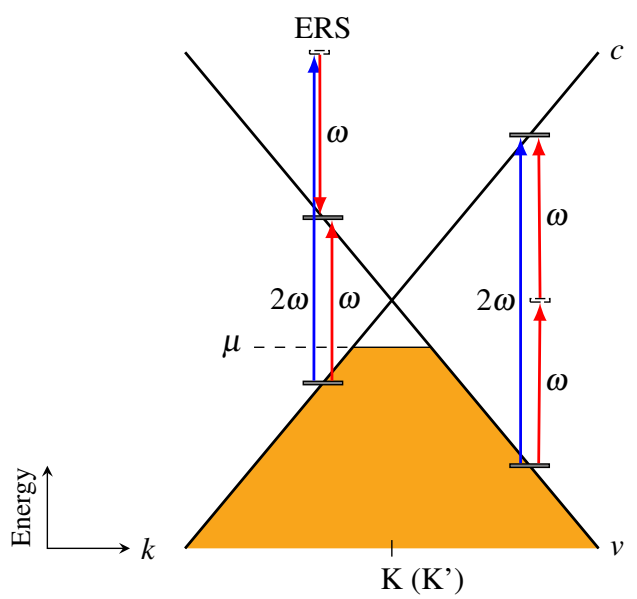

Figure 2. The conventional excitation scheme (right) employs interference between two-photon absorption at $\omega$ (red arrows) and onephoton absorption at $2 \omega$ (blue arrows). The additional contribution at $\hbar \omega$ (left) occurs when one-photon absorption at $\omega$ interferes with stimulated electronic Raman scattering (ERS, $2 \omega$ absorption and $\omega$ emission). Depending on the chemical potential $\mu$, the ERS contribution is allowed $(2|\mu|<\omega)$ or blocked $(2|\mu|>\omega$, not shown).

$$
\begin{aligned}
& \eta_{I}^{\prime a b c d}(\omega)=\frac{\pi i e^{4}}{\hbar^{3} \omega^{3}} \sum_{c, v} \int \frac{\mathrm{d}^{2} k}{4 \pi^{2}}\left[v_{c c}^{a}(\mathbf{k})-v_{v v}^{a}(\mathbf{k})\right] \\
& \quad \times\left[v_{c v}^{b *}(\mathbf{k}) w_{c v}^{\prime c d}(\mathbf{k})+v_{c v}^{c *}(\mathbf{k}) w_{c v}^{\prime b d}(\mathbf{k})\right] \delta\left[\omega_{c v}(\mathbf{k})-\omega\right] .
\end{aligned}
$$

For a three-dimensional material, the integration over reciprocal space in Eqs. (2), (7), (9), (10) and (11) should read $\int \mathrm{d}^{3} k / 8 \pi^{3}$.

\section{ANISOTROPIC PHOTOINDUCED CURRENT INJECTION IN GRAPHENE}

We consider graphene to illustrate the two-color interference effect at $\hbar \omega$ in experimentally accessible conditions. The part of reciprocal space relevant for optical response are the two valleys near $K$ and $K^{\prime}$ at the vertices of the hexagonal Brillouin zone, where the electrons follow a Dirac-like spectrum [Fig. 2]. The gapless band dispersion has the advantage that a bandgap energy is not a limiting factor for the occurrence of absorption at $\hbar \omega$. Instead, the chemical potential $\mu$ plays the role of the limiting factor. Photocurrent generation via $\mathrm{CC}$ includes the contribution from both interference channels presented in the previous section and illustrated in Fig. 2 for graphene. Experimentally, electrically tuning the chemical potential allows one to enter and exit the regime where absorption at $\hbar \omega$ occurs. It is thus possible to identify the ERS contribution.

The diagonalized model Hamiltonian and corresponding velocity operator near the K point of graphene take the form ${ }^{16}$

$$
H_{0} \rightarrow \hbar v_{F}\left(\begin{array}{cc}
k & 0 \\
0 & -k
\end{array}\right), \quad \mathbf{v} \rightarrow v_{F}\left(\begin{array}{cc}
\hat{k} & i \hat{\phi} \\
-i \hat{\phi} & -\hat{k}
\end{array}\right),
$$


where $v_{F}$ is the Fermi velocity, $\mathbf{k}=k \hat{k}$ is the in-plane crystal momentum relative to the Dirac point, and $\hat{\phi}=\hat{z} \times \hat{k}$ is an inplane unit vector perpendicular to $\mathbf{k}$. The treatment for the $\mathrm{K}$ ' valley is analogous and its presentation neglected for the remainder of the paper as it simply introduces a degeneracy factor of two, in addition to the spin degeneracy factor of two.

The generation of a photocurrent via CC is best illustrated by the unbalanced carrier distributions in reciprocal space due to the interference effect being constructive or destructive at different wavevectors. ${ }^{6}$ The carrier distributions due to 2PA and 1PA at $2 \omega$ have been previously calculated. ${ }^{16}$ Here we present the result of the ERS contribution and its interference with 1PA at $\omega$. The ERS has previously been calculated in the context of conventional Raman spectroscopy. ${ }^{27}$

The distribution of photoexcited carriers in reciprocal space due to the two-color field, $\dot{n}=\dot{n}(2 \omega ; \mathbf{k})+\dot{n}(\omega ; \mathbf{k})$, is shown in Fig. 3, taking both the conventional and ERS contributions to be equally allowed, e.g. for intrinsic graphene. The distribution due to conventional $\mathrm{CC}$ is

$$
\dot{n}(2 \omega ; \mathbf{k})=\left|\Omega_{c v}^{(1)}(2 \omega ; \mathbf{k})+\Omega_{c v}^{(2)}(\omega ; \mathbf{k})\right|^{2}
$$

and occurs on the (outer) circle of radius $k=\omega / v_{F}$; the distribution due to ERS-induced CC is

$$
\dot{n}(\omega ; \mathbf{k})=\left|\Omega_{c v}^{(1)}(\omega ; \mathbf{k})+\Omega_{c v}^{(2)}(2 \omega,-\omega ; \mathbf{k})\right|^{2}
$$

and occurs on the (inner) circle of radius $k=\omega / 2 v_{F}$.

For circular polarization [Fig. 3(a,b)] the distributions depend on the angle $\phi_{k}=\tan ^{-1}\left(k_{y} / k_{x}\right)$ and the CC parameter $\Delta \varphi \equiv 2 \varphi_{\omega}-\varphi_{2 \omega}$ relating the phases of the two frequency components. For opposite-circularly polarized $\omega$ and $2 \omega$ beams, $\hat{\mathbf{e}}_{2 \omega}=-\hat{\mathbf{e}}_{\omega}=\sigma^{ \pm}$, the distributions for the carrier injection with net energy absorption of $2 \hbar \omega$ and $\hbar \omega$ vary as $\dot{n}(2 \omega ; \mathbf{k}) \propto 1-\sin \left(\Delta \varphi \mp 3 \phi_{k}\right)$ and $\dot{n}(\omega ; \mathbf{k}) \propto \frac{3}{4}(1+\sin (\Delta \varphi \pm$ $\left.3 \phi_{k}\right)$ ), respectively, neither of which yield a current. For co-circularly polarized beams, $\hat{\mathbf{e}}_{2 \omega}=\hat{\mathbf{e}}_{\omega}=\sigma^{ \pm}$, the distributions of $\dot{n}(2 \omega ; \mathbf{k}) \propto 1+\sin \left(\Delta \varphi \pm \phi_{k}\right)$ and $\dot{n}(\omega ; \mathbf{k}) \propto \frac{3}{4}(1-$ $\left.\sin \left(\Delta \varphi \mp \phi_{k}\right)\right)$ yield opposite current contributions, although a net current injection remains. The distributions are plotted for $\Delta \varphi=\pi / 2$.

For linearly-polarized beams [Fig. 3(c-f)], without loss of generality the polarization axis $\hat{\mathbf{e}}_{2 \omega}$ is chosen along $\hat{x}$ and the k-dependent carrier distributions follow

$$
\begin{aligned}
\dot{n}(2 \omega ; \mathbf{k}) & \propto\left|\sin \left(\phi_{k}\right)+i e^{-i \Delta \varphi} \sin \left(2 \phi_{k}-2 \theta\right)\right|^{2} \\
\dot{n}(\omega ; \mathbf{k}) & \propto\left|\sin \left(\phi_{k}-\theta\right)-\frac{i}{4} e^{-i \Delta \varphi}\left[\sin \left(2 \phi_{k}-\theta\right)+3 \sin (\theta)\right]\right|^{2}
\end{aligned}
$$

where $\theta$ is the angle between the polarization axes $\hat{\mathbf{e}}_{\omega}$ and $\hat{\mathbf{e}}_{2 \omega}$. The first term in each of Eqs. (15) and (16) is due to 1PA, and the second term is due to 2PA [Eq. (15)] or stimulated ERS [Eq. (16)]. The distributions are shown for $\Delta \varphi=\pi / 2$ and the polarization axis $\hat{\mathbf{e}}_{\omega}$ forming angles of $\theta=0,45^{\circ}, 90^{\circ}$, and $135^{\circ}$ with respect to $\hat{\mathbf{e}}_{2 \omega}$. For parallel and perpendicular polarization, the current due to the ERS contribution opposes the
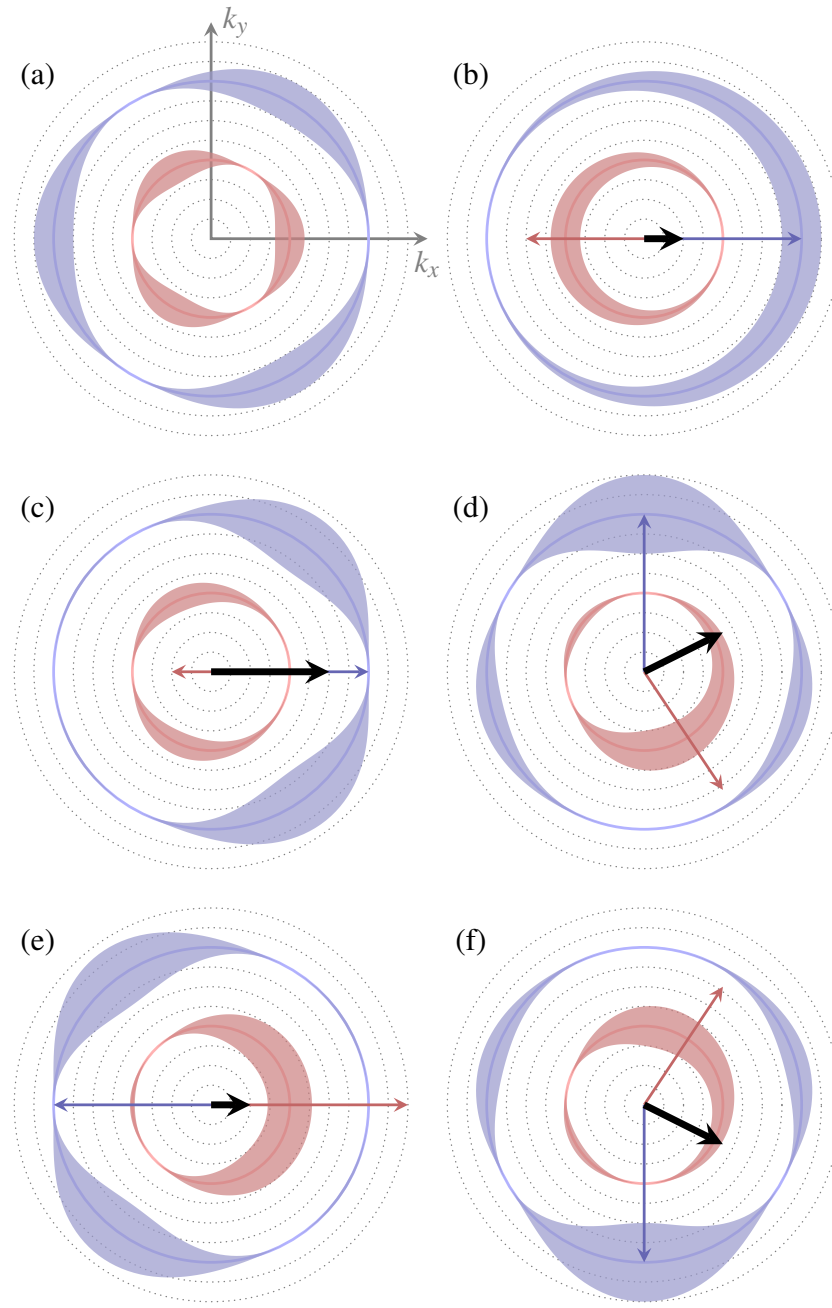

Figure 3. Reciprocal space distribution of injected carriers in intrinsic graphene under two-color, $\omega$ and $2 \omega$ field. Absorption processes up to second order in the field are considered, with net energy absorption of $\hbar \omega$ (red, inner distribution) and $2 \hbar \omega$ (blue, outer distribution), respectively due to ERS-1PA interference and 1PA-2PA interference. The carrier injection rates [Eq. (13) and Eq. (14)] are indicated by the thickness of the distributions, the current resulting from each distribution is indicated by a correspondingly colored arrow, and the overall current injection is indicated by a thick, black arrow. Photoresponse for circularly-polarized $\omega$ and $2 \omega$ beams: (a) opposite polarization, yielding no net current injection, and (b) cocircular polarization, yielding a net current injection. Photoresponse for linearly-polarized $\omega$ and $2 \omega$ beams: (c) $\theta=0$, i.e. parallel polarization axes, (d) $\theta=\pi / 4$, (e) $\theta=\pi / 2$, i.e. perpendicular polarization axes, and (f) $\theta=3 \pi / 4$; all yielding current injection.

current due to the conventional injection process; in the latter case, the ERS contribution is strong enough to cause the direction of the net current to flip. For $45^{\circ}$ and $135^{\circ}$, the ERS contribution adds a current component along the $\hat{\mathbf{e}}_{2 \omega}$ direction, while its component along $\hat{\mathbf{e}}_{2 \omega}^{\perp}$ opposes the conventional injection process, although not sufficiently to flip the net current along this direction. The magnitude of the photocurrent is anisotropic in $\theta$ due to the ERS contribution. 
The symmetry of graphene yields $\eta_{I}^{x x x x}, \eta_{I}^{x y y x}$, and $\eta_{I}^{x y x y}=$ $\eta_{I}^{x x y y}$ as independent components of the current injection tensor; in addition, the relation $2 \eta_{I}^{x y x y}=\eta_{I}^{x x x x}-\eta_{I}^{x y y x}$ holds since an isotropic band model is considered. The disparity parameter $d=\eta_{I}^{x y y x} / \eta_{I}^{x x x x}$ characterizes the sensitivity of the current injection to the angle $\theta$ between polarization axes for linearlypolarized $\omega$ and $2 \omega$ beams; different values of $d$ and $\theta$ lead to injected currents with different magnitude and direction. ${ }^{16}$ The additional current injection term presented in this paper yields the following implications for graphene. In the conventional regime [Eq. (2)], the nonzero current injection tensor components were given by

$$
\eta_{I}^{x x x x}=\eta_{I}^{x y x y}=\eta_{I}^{x x y y}=-\eta_{I}^{x y y x}=i \bar{\eta}_{I}(\omega),
$$

where

$$
\bar{\eta}_{I}(\omega) \equiv g_{s} g_{v} e^{4} v_{F}^{2}(2 \hbar \omega)^{-3},
$$

with $g_{s}=g_{v}=2$ describing spin and valley degeneracy, respectively. ${ }^{16}$ As a result of this contribution, a current component is injected with effective parameter $d_{\text {eff }}=-1$ and the magnitude of this current is $\theta$-independent. The ERS contribution, in contrast, is strongly $\theta$-dependent: taken on its own it contributes an effective parameter $d_{\mathrm{eff}}=-5$, meaning that it yields a current whose magnitude is five times stronger when the beam polarization axes are perpendicular $(\theta=\pi / 2)$ compared to them being parallel $(\theta=0)$. The nonzero tensor components for this contribution [Eq. (11)] are given by

$$
\eta_{I}^{\prime x x x x}=\frac{1}{3} \eta_{I}^{\prime x y x y}=\frac{1}{3} \eta_{I}^{\prime x x y y}=-\frac{1}{5} \eta_{I}^{\prime x y y x}=-\frac{1}{4} i \bar{\eta}_{I}(\omega) .
$$

This contribution doesn't occur if the transition at $\omega$ is blocked due to the lower level being depleted or the upper level occupied. For intrinsic graphene the two discussed interference channels are equally allowed, the resulting current injection tensor includes both contributions, its components are given by

$$
\eta_{I}^{x x x x}=3 \eta_{I}^{x y x y}=3 \eta_{I}^{x x y y}=3 \eta_{I}^{x y y x}=\frac{3}{4} i \bar{\eta}_{I}(\omega),
$$

and the corresponding parallel-perpendicular polarization disparity parameter is $d=1 / 3$. This value is closer to a typical semiconductor such as GaAs, which has $|d| \approx 0.2$ for a fundamental photon energy of about $1 \mathrm{eV}$ (below the onset of 1PA at $\omega) .{ }^{24}$ For intrinsic graphene the parameter $d$ is independent of the light frequency, as it would be were the ERS contribution not included. ${ }^{16}$

Let us now consider the effect of a finite temperature and nonzero chemical potential on the current injection. The deviation from the semiconductor vacuum state results in the Pauli blocking of optical transitions, as observed in linear absorption. ${ }^{28}$ Since the two contributions to current injection, Eq. (2) and Eq. (11), occur at different excitation energies, it is possible to partly block the lower-energy transition while retaining the higher-energy one. This allows one to tune the polarization sensitivity of the resulting current. For a $p$-doped sample, if $f_{\omega}$ is the population of electrons as a function of the energy level $\hbar \omega$ relative to the Dirac point, then the disparity

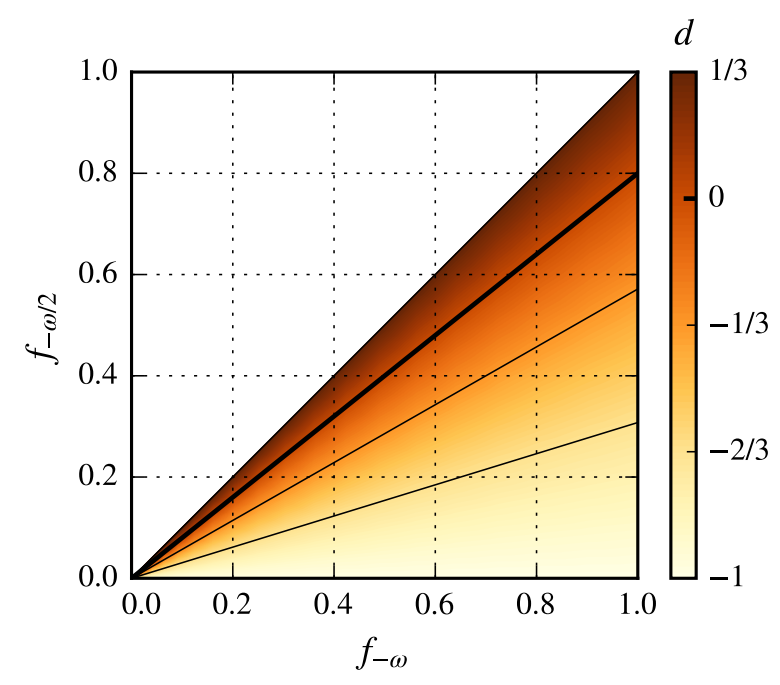

Figure 4. Contour plot of the parallel-perpendicular polarization disparity parameter $d=\eta_{I}^{x y y x} / \eta_{I}^{x x x x}$ in $p$-doped graphene as a function of the electron population $f_{-\omega}$ and $f_{-\omega / 2}$ in the valence band $\left(f_{-\omega / 2} \leq f_{-\omega}\right)$. With $f_{-\omega / 2}=0$, stimulated ERS and 1PA at $\omega$ is blocked and interference of 2PA and 1PA at $2 \omega$ yields $d=-1$ characterizing an injection current that is isotropic with respect to $\theta=\cos ^{-1}\left(\hat{\mathbf{e}}_{\omega} \cdot \hat{\mathbf{e}}_{2 \omega}\right)$. With $f_{-\omega / 2} \neq 0$, the additional ERS contribution yields $d>-1$ characterizing an anisotropic current. In particular, for $d=0$ (thick contour line) the injection current vanishes for perpendicular polarization axes, $\theta=\pi / 2$.

parameter is

$$
d=-\frac{4 f_{-\omega}-5 f_{-\omega / 2}}{4 f_{-\omega}-f_{-\omega / 2}} .
$$

This expression is plotted in Fig. 4, and shows that the allowed values are in the range $-1 \leq d \leq 1 / 3$. Taking $f_{-\omega / 2}=0$ yields $d=-1$ and corresponds to the previous result with complete blocking of the linear absorption of the fundamental due to the lower level being depleted, ${ }^{16}$ while the ratio of $f_{-\omega / 2} / f_{-\omega}=1$ yields $d=1 / 3$ and corresponds to the situation presented in Fig. 3. For $d>0$, the injected current in the cross-polarized configuration flips direction due to the ERS contribution. A value of $d=0$ (thick contour line in Fig. 4) corresponds to the complete suppression of the current injection for perpendicular polarization axes; this is achieved by adjusting the population to the ratio of $f_{-\omega / 2} / f_{-\omega}=0.8$. Thus, a range of polarization-sensitive and polarization-insensitive configurations are available.

The variation of the current injection characteristics according to temperature and nonzero chemical potential affects our understanding of previous experiments in multilayer epitaxial graphene, ${ }^{15,17,18}$ as the contribution from a single layer depends on its doping level. The doping level in multilayer epitaxial graphene decreases exponentially from $365 \mathrm{meV}$ for the layer closest to the substrate to zero doping for the top layers, with a charge screening length of one layer. ${ }^{15}$ The total current injection across all layers is made up of an isotropic contribution from the few heavily-doped layers near the substrate $(d=-1)$ and an anisotropic contribution from the remaining 


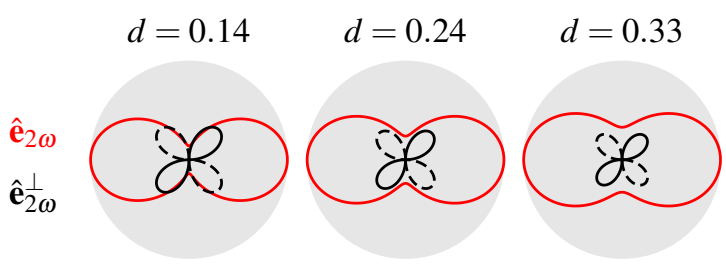

Figure 5. Angular plots of the current injection strength as a function of the angle $\theta$ between the linear polarization axes $\hat{\mathbf{e}}_{\omega}$ and $\hat{\mathbf{e}}_{2 \omega}$, for the ratio $d=\eta_{I}^{x y y x} / \eta_{I}^{x x x x}$ taking values of $0.14,0.24$, and 0.33 . The current components along $\hat{\mathbf{e}}_{2 \omega}$ (plain red line) and $\hat{\mathbf{e}}_{2 \omega}^{\perp}$ (plain/dashed black line) are plotted; a dashed line indicates a negative current along that axis.

mostly undoped layers $(d=1 / 3)$. In the original experiment the incoming beams were cross-linearly polarized; ${ }^{15}$ taking the ERS contribution into account yields counter-propagating currents in the heavily-doped and undoped layers, as illustrated in Fig. 3(e). A subsequent experiment resolved the angular dependence on the polarization axes and found that it did not correspond to the simple model of a single graphene layer. ${ }^{17}$ In both experiments the current injection was detected via the emitted $\mathrm{THz}$ radiation with the signal collected behind the sample. Light attenuation is stronger for the $\mathrm{THz}$ signal than for the frequencies of the incoming beams. Thus, although there are only a few heavily-doped layers, they could contribute relatively strongly to the detected $\mathrm{THz}$ signal due to their proximity with the detector. It is realistic to expect the signal to have a value of $d$ in the range 0.14 to 0.24 ; the resulting polarization dependence is shown in Fig. 5, and is in good agreement with the polarization-resolved experimental data. ${ }^{17}$ Thus the inclusion of the Raman term identified here could lead to an understanding of the experimental results even without the assumption of interlayer coupling.

\section{CONCLUSIONS}

We have presented the complete current response of the gapless semiconductor graphene due to coherent control of light at frequencies $\omega$ and $2 \omega$. By considering the additional interference effect between stimulated ERS and 1PA of the fundamental, occurring with a net energy absorption of $\hbar \omega$, we have shown that graphene presents a photoresponse sensitive to the relative orientation of the linear polarization axes of the light. This polarization sensitivity, characterized by a tunable disparity parameter $-1 \leq d \leq 1 / 3$, is in stark contrast with the polarization-isotropic photocurrent contribution due to 1PA and 2PA interference alone. ${ }^{16}$ The value of $d=1 / 3$ for intrinsic graphene is closer to typical values in GaAs, ${ }^{24}$ and with a value of $d=0$ it is possible to completely suppress the current injection for perpendicular polarization axes.
Thus, with its Fermi level adjustable through doping or electrical gating, graphene offers tunable, polarization sensitive applications.

Although the excitation mechanism presented here differs from the excitation mechanisms of previous studies, it is expected that the subsequent dynamics follows a similar course. The injected photocurrent is limited by momentum relaxation and is expected to decay following excitation. For a pulsed laser source, it has been reported that hot carriers reach an isotropic distribution $150 \mathrm{fs}$ to $250 \mathrm{fs}$ after excitation, with intraband carrier-phonon scattering being the main momentum relaxation mechanism. ${ }^{18,29-33}$ On shorter timescales, collinear carrier-carrier scattering preserves the anisotropy, ${ }^{18,32,33}$ allowing for similar photocurrents to be detected..$^{15,17,18}$

While the additional interference term between stimulated ERS and 1PA is correctly included in a recent treatment of third-order nonlinear optical conductivities of doped graphene, ${ }^{22}$ it had not been carefully considered in previous studies of coherent optical injection and control in graphene and carbon nanotubes. ${ }^{12-18}$ However, we have shown that this term modifies the CC photocurrent response significantly, raising the question whether previous experimental results need to be reinterpreted. ${ }^{14,15,17,18}$ In experiments with fixed polarization the ERS contribution does not play a crucial role, specifically the conclusions of Newson et al. regarding CC using colinearly-polarized beams in carbon nanotubes, ${ }^{14}$ and those of Sun et al. using cross-polarized beams in multilayer epitaxial graphene, ${ }^{15,18}$ are not altered. Considering the ERS contribution and depending on the level of doping, crosspolarized beams could lead to counter-propagating currents in the undoped and heavily-doped layers of epitaxial graphene, an effect that is not resolvable in the employed $\mathrm{THz}$ detection technique. Such a different response from the undoped and heavily-doped layers offers an alternative explanation for the polarization-sensitive data of Sun et al. [17], which the authors attributed to interlayer coupling.

Besides gapless semiconductors such as graphene, a careful treatment of the interference between stimulated electronic Raman scattering and linear absorption is important for the complete description of two-color coherent control in metals, topological insulators, and in semiconductors when $\hbar \omega$ exceeds the bandgap. Calculations like the one we presented will play an important role in the analysis of experiments involving those systems.

\section{ACKNOWLEDGMENTS}

This work was supported by the Deutsche Forschungsgemeinschaft (DFG) under project number SFB 767 and the Konstanz Center for Applied Photonics (CAP). J.E. S. acknowledges funding from the National Sciences and Engineering Research Council (NSERC) of Canada. The authors acknowledge useful discussions with Jin Luo Cheng, Dong Sun and Ted Norris. 
1 P. Král, I. Thanopulos, and M. Shapiro, "Colloquium: Coherently controlled adiabatic passage," Rev. Mod. Phys. 79, 53 (2007).

2 P. Brumer and M. Shapiro, "Control of unimolecular reactions using coherent light," Chem. Phys. Lett. 126, 541 (1986); M. Shapiro, J. W. Hepburn, and P. Brumer, "Simplified laser control of unimolecular reactions: Simultaneous $\left(\omega_{1}, \omega_{3}\right)$ excitation," ibid., 149, 451 (1988); M. Shapiro and P. Brumer, "Coherent control of molecular dynamics," Rep. Prog. Phys. 66, 859 (2003).

3 W. S. Warren, H. Rabitz, and M. Dahleh, "Coherent control of quantum dynamics: The dream is alive," Science 259, 1581 (1993); H. Rabitz, R. de Vivie-Riedle, M. Motzkus, and K. Kompa, "Whither the future of controlling quantum phenomena?" ibid., 288, 824 (2000).

4 E. Dupont, P. B. Corkum, H. C. Liu, M. Buchanan, and Z. R. Wasilewski, "Phase-controlled currents in semiconductors," Phys. Rev. Lett. 74, 3596 (1995).

5 R. Atanasov, A. Haché, J. L. P. Hughes, H. M. van Driel, and J. E. Sipe, "Coherent control of photocurrent generation in bulk semiconductors," Phys. Rev. Lett. 76, 1703 (1996); A. Haché, Y. Kostoulas, R. Atanasov, J. L. P. Hughes, J. E. Sipe, and H. M. van Driel, "Observation of coherently controlled photocurrent in unbiased, bulk GaAs," ibid., 78, 306 (1997).

6 M. Sheik-Bahae, "Quantum interference control of current in semiconductors: Universal scaling and polarization effects," Phys. Rev. B 60, R11257 (1999).

7 E. A. Manykin, "Quantum interference and coherent control," Laser Phys. 11, 60 (2001).

8 D. H. Marti, M.-A. Dupertuis, and B. Deveaud, "General theory for the interference of two-photon and one-photon processes in semiconductor heterostructures," Ann. Phys. 316, 234 (2005).

9 S. G. Rodrigo, H. Harutyunyan, and L. Novotny, "Coherent control of light scattering from nanostructured materials by secondharmonic generation,” Phys. Rev. Lett. 110, 177405 (2013).

10 S. Thunich, C. Ruppert, A. W. Holleitner, and M. Betz, "Subdiffraction optical coherent control of ultrafast electrical currents in antenna devices on GaAs," Appl. Phys. Lett. 101, 251119 (2012).

11 C. Zhuang, C. R. Paul, X. Liu, S. Maneshi, L. S. Cruz, and A. M. Steinberg, "Coherent control of population transfer between vibrational states in an optical lattice via two-path quantum interference," Phys. Rev. Lett. 111, 233002 (2013); preprint at arXiv:1207.6427.

12 P. Král and D. Tománek, "Laser-driven atomic pump," Phys. Rev. Lett. 82, 5373 (1999).

13 E. J. Mele, P. Král, and D. Tománek, "Coherent control of photocurrents in graphene and carbon nanotubes," Phys. Rev. B 61, 7669 (2000); preprint at arXiv:cond-mat/9911151.

14 R. W. Newson, J.-M. Ménard, C. Sames, M. Betz, and H. M. van Driel, "Coherently controlled ballistic charge currents injected in single-walled carbon nanotubes and graphite," Nano Lett. 8, 1586 (2008).

15 D. Sun, C. Divin, J. Rioux, J. E. Sipe, C. Berger, W. A. de Heer, P. N. First, and Th. B. Norris, "Coherent control of ballistic photocurrents in multilayer epitaxial graphene using quantum interference," Nano Lett. 10, 1293 (2010).

16 J. Rioux, G. Burkard, and J. E. Sipe, "Current injection by coherent one- and two-photon excitation in graphene and its bilayer," Phys. Rev. B 83, 195406 (2011); preprint at arXiv:1101.4030.

17 D. Sun, J. Rioux, J. E. Sipe, Y. Zou, M. T. Mihnev, C. Berger, W. A. de Heer, P. N. First, and Th. B. Norris, "Evidence for interlayer electronic coupling in multilayer epitaxial graphene from polarization-dependent coherently controlled photocurrent generation," Phys. Rev. B 85, 165427 (2012); preprint at arXiv:1109.2850.

18 D. Sun, C. Divin, M. T. Mihnev, T. Winzer, E. Malić, A. Knorr, J. E. Sipe, C. Berger, W. A. de Heer, P. N. First, and Th. B. Norris, "Current relaxation due to hot carrier scattering in graphene," New J. Phys. 14, 105012 (2012).

19 P. A. Wolff, "Thomson and Raman scattering by mobile electrons in crystals," Phys. Rev. Lett. 16, 225 (1966).

20 F. Comas, C. T. Giner, and R. Perez-Alvarez, "Interbandintraband electronic Raman scattering in semiconductors," J. Phys. C 19, 6479 (1986).

21 T. P. Devereaux and R. Hackl, "Inelastic light scattering from correlated electrons,” Rev. Mod. Phys. 79, 175 (2007); preprint at arXiv:cond-mat/0607554.

22 J. L. Cheng, N. Vermeulen, and J. E. Sipe, "Third order optical nonlinearity of graphene," New J. Phys. 16, 053014 (2014).

23 R. A. Muniz and J. E. Sipe, "Coherent control of optical injection of spin and currents in topological insulators," Phys. Rev. B 89, 205113 (2014); preprint at arXiv:1401.1241.

24 J. Rioux and J. E. Sipe, "Optical injection processes in semiconductors," Physica E 45, 1 (2012).

25 M. Sheik-Bahae, D. C. Hutchings, D. J. Hagan, and E. W. V. Stryland, "Dispersion of bound electronic nonlinear refraction in solids," IEEE J. Quantum Electron. 27, 1296 (1991); M. SheikBahae, J. Wang, R. DeSalvo, D. J. Hagan, and E. W. V. Stryland, "Measurement of nondegenerate nonlinearities using a twocolor Z scan," Opt. Lett. 17, 258 (1992); M. Sheik-Bahae and M. P. Hasselbeck, "Third-order optical nonlinearities," in Handbook of Optics, Vol. 4, Fiber Optics and Nonlinear Optics, edited by M. Bass (McGraw-Hill, New York, 2001) 2nd ed., Chap. 17.

26 M. J. Stevens, R. D. R. Bhat, J. E. Sipe, H. M. van Driel, and A. L. Smirl, "Coherent control of carrier population and spin in (111)-GaAs," Phys. Status Solidi B 238, 568 (2003).

27 H.-Y. Lu and Q.-H. Wang, "Electronic Raman scattering in graphene," Chin. Phys. Lett. 25, 3746 (2008).

28 K. F. Mak, M. Y. Sfeir, Y. Wu, C. H. Lui, J. A. Misewich, and T. F. Heinz, "Measurement of the optical conductivity of graphene," Phys. Rev. Lett. 101, 196405 (2008); preprint at arXiv:0810.1269.

29 J. M. Dawlaty, S. Shivaraman, M. Chandrashekhar, F. Rana, and M. G. Spencer, "Measurement of ultrafast carrier dynamics in epitaxial graphene," Appl. Phys. Lett. 92, 042116 (2008); preprint at arXiv:0712.0119.

30 M. Breusing, S. Kuehn, T. Winzer, E. Malić, F. Milde, N. Severin, J. P. Rabe, C. Ropers, A. Knorr, and T. Elsaesser, "Ultrafast nonequilibrium carrier dynamics in a single graphene layer," Phys. Rev. B 83, 153410 (2011).

31 S. Winnerl, M. Orlita, P. Plochocka, P. Kossacki, M. Potemski, T. Winzer, E. Malić, A. Knorr, M. Sprinkle, C. Berger, W. A. de Heer, H. Schneider, and M. Helm, "Carrier relaxation in epitaxial graphene photoexcited near the Dirac point," Phys. Rev. Lett. 107, 237401 (2011); preprint at arXiv:1105.2518.

32 D. Brida, A. Tomadin, C. Manzoni, Y. J. Kim, A. Lombardo, S. Milana, R. R. Nair, K. S. Novoselov, A. C. Ferrari, G. Cerullo, and M. Polini, "Ultrafast collinear scattering and carrier multiplication in graphene," Nat. Commun. 4, 1987 (2013); preprint at arXiv:1209.5729.

33 M. Mittendorff, T. Winzer, E. Malić, A. Knorr, C. Berger, W. A. de Heer, H. Schneider, M. Helm, and S. Winnerl, "Anisotropy of excitation and relaxation of photogenerated charge carriers in graphene," Nano Lett. 14, 1504 (2014); preprint at arXiv:1312.5860. 